\title{
Reincorporación de trabajadores recuperados COVID-19 y COVID persistente en la industria metalmecánica en Lima Perú 2021 \author{
metalworking industry in Lima Peru 2021
} \\ Reincorporation of recovered workers COVID-19 and long COVID in the
}

\author{
https://doi.org/10.52808/bmsa.7e5.613.014 \\ Roberto Carlos Dávila Morán ${ }^{1, *}$ \\ https://orcid.org/0000-0003-3181-8801 \\ Eucaris del Carmen Agüero Corzo ${ }^{2}$ \\ https://orcid.org/0000-0003-4587-3852 \\ Leonardo Velarde Dávila ${ }^{3}$ \\ https://orcid.org/0000-0002-8096-0196 \\ Héctor Portillo Rios ${ }^{4}$ \\ https://orcid.org/0000-0003-1432-8472 \\ José Leonor Ruiz Nizama ${ }^{5}$ \\ https://orcid.org/0000-0001-8935-5366 \\ Carlos Enrique Guanilo Paredes 6 \\ https://orcid.org/0000-0003-0444-244X \\ Félix Román Guillén Pedraza ${ }^{4}$ \\ https://orcid.org/0000-0002-5528-4026
}

Recibido: $15 / 04 / 2021$

Aceptado: 01/08/2021

\section{RESUMEN}

El COVID persistente se caracteriza por síntomas y secuelas funcionales y psicológicas que persisten por más de 12 semanas post infección, tales como: fatiga, disnea, ansiedad, depresión; generando inconvenientes en la reanudación de actividades laborales de los trabajadores. Se realizó un estudio epidemiológico descriptivo de corte transversal, evaluando la reincorporación de trabajadores operadores de industrias metalmecánicas de Lima, Perú; recuperados y COVID persistentes, entre el tercer trimestre 2020 y primer trimestre 2021. Se aplicaron las escalas de disnea de Borg y la escala de disnea modificada del Medical Research Council (mMRC), además de la escala de ansiedad y depresión de Goldberg. Los resultados se analizaron mediante estadística descriptiva, empleando medidas de tendencia central y dispersión. Se observó que más del $80 \%$ de trabajadores recuperados son reincorporados; y para COVID persistente se reincorpora entre $75 \%$ y $43 \%$ para los cargos 8121 y 8122 , respectivamente. El esfuerzo respiratorio en reposo resultó normal por la escala de Borg para cada grupo, mientras que en esfuerzo se obtuvo un valor $\mathrm{R}^{2}=0.2986$ indicando la no reincorporación en $74,51 \%$ y $43,33 \%$ en las categorias 8121 y 8122 , respectivamente; además $45,12 \%$ y $42,68 \%$ de los trabajadores recuperados y COVID persistentes mostraron sintomas de fatiga; $36,25 \%$ y $35,26 \%$ de ansiedad; $25,4 \%$ y $15,21 \%$ de depresión, respectivamente; asimismo, el total de trabajadores presentaron sobrepeso con IMC promedio $>26 \mathrm{Kg} / \mathrm{m}^{2}$; siendo más elevado en mujeres $\left(27,4 \mathrm{Kg} / \mathrm{m}^{2}\right)$. El COVID-19 afecta la salud de trabajadores (recuperados y COVID persistentes), reduce el reintegro al trabajo y en consecuencia la productividad de las empresas.

Palabras clave: COVID persistente, fatiga, disnea, depresión.

\section{ABSTRACT}

Persistent COVID is characterized by functional and psychological symptoms and sequelae that persist for more than 12 weeks post infection, such as: fatigue, dyspnea, anxiety, depression; generating inconveniences in the resumption of work activities of workers. A descriptive, cross-sectional epidemiological study was carried out, evaluating the reincorporation of workers operating in metalworking industries in Lima, Peru; recovered and persistent COVID, between the third quarter 2020 and the first quarter 2021. The Borg dyspnea scales and the modified dyspnea scale of the Medical Research Council ( $m M R C)$ were applied, in addition to the Goldberg anxiety and depression scale. The results were analyzed using descriptive statistics, using measures of central tendency and dispersion. It was observed that more than $80 \%$ of recovered workers are reinstated; and for persistent COVID, between $75 \%$ and $43 \%$ are reinstated for charges 8121 and 8122, respectively. Respiratory effort at rest was normal by the Borg scale for each group, while in effort a value $R 2=0.2986$ was obtained, indicating no reincorporation in $74.51 \%$ and $43.33 \%$ in categories 8121 and 8122 , respectively; also $45.12 \%$ and $42.68 \%$ of the recovered workers and persistent COVID showed symptoms of fatigue; $36.25 \%$ and $35.26 \%$ anxiety; $25.4 \%$ and $15.21 \%$ of depression, respectively; Likewise, the total of workers were overweight with an average BMI> $26 \mathrm{Kg} / \mathrm{m} 2$; being higher in women (27.4 Kg / $\mathrm{m} 2$ ). COVID-19 affects the health of workers (recovered and persistent COVID), reduces return to work and consequently the productivity of companies.

1 Universidad Privada del Norte (UPN). Lima, Perú 2 Universidad Pedagógica Experimental Libertador (UPEL). Maturín, Venezuela 3 Universidad de San Martín de Porres (USMP). Lima, Perú 4 Universidad Inca Garcilaso de la Vega (UIGV). Lima, Perú 5 Universidad Nacional del Callao (UNAC). Callao, Perú 


\section{Introducción}

Desde el inicio de la pandemia por COVID-19, existen más de 160 millones de casos confirmados y la mayoría de los pacientes experimentan enfermedad leve o moderada, con un tiempo promedio de recuperación de 2-3 semanas dependiendo de la gravedad de los síntomas. Sin embargo, independientemente de la gravedad de la infección aguda, 1 de cada 10 personas puede presentar síntomas durante 12 semanas o más. En tal sentido, el Instituto para la atención y cuidado de excelencia en salud (National Institute for Health and Care Excellence guideline por sus siglas en inglés) establece que el COVID prolongado o persistente comprende el COVID-19 sintomático continuo (síntomas durante 4-12 semanas) y el síndrome post-COVID-19 (persistencia de síntomas durante más de 12 semanas en ausencia de un diagnóstico alternativo) (Aiyegbusi et al., 2021).

Diversos estudios han hecho referencia a la evolución y los principales síntomas presentados por pacientes COVID. En ese sentido, en el $2^{\circ}$ Congreso Nacional Multidisciplinar Covid-19, reportaron datos de una encuesta internacional, encontrando que en $96 \%$ de los encuestados el promedio de persistencia de los síntomas de COVID fue de 90 días, con afectación multiorgánica y multisistémica, siendo los más frecuentes la fatiga, malestar post esfuerzo, síntomas psicológicos, digestivos, respiratorios y neurológicos; destacando la disfunción cognitiva y del aparato locomotor (Riesco et al., 2021). Por su parte, la sociedad española de médicos generales y de familia señaló en un estudio que $81,79 \%$ de personas encuestadas, presentaban síntomas compatibles de COVID-19 persistente, con evolución de más de 185 días (más de 6 meses) caracterizados por cansancio, astenia, malestar general, cefalea, mialgias, disnea, artralgias, falta de concentración, dolor de espalda, presión en el pecho, ansiedad, fallos de memoria, cervicalgia, diarrea, dolor torácico, palpitaciones, mareos, parestesias; además exhibieron afectación del área neurológica, psicológica, emocional, aparato locomotor, respiratorio, alteraciones digestivas y cardíacas. Asimismo, la mitad de los encuestados refirieron incapacidad notable para realizar actividades de la vida diaria como el aseo personal, actividades de casa y trabajar fuera de casa (SEMG, 2021).

En tal sentido, las alteraciones físicas y psicológicas, asociadas a la infección por COVID-19 o COVID persistente, pueden resultar en dificultad para la reincorporación al trabajo, puesto que es posible que a los afectados les cueste realizar sus actividades cotidianas, pero que tenga que trabajar por razones económicas o sociales, por el bien de su salud mental. La pandemia también ha afectado a los empresarios, quienes han cambiado la organización del trabajo para lograr un entorno seguro para los trabajadores y los clientes y además han presenciado cómo un gran número de sus trabajadores se ausentaban por la enfermedad (Macdonald et al., 2021).

En efecto, el impacto del COVID-19 en el mundo laboral ha conllevado a la pérdida y precarización de los empleos, puesto que los gobiernos de todo el mundo reaccionaron con diferente tiempo de respuesta y medidas como la cuarentena y el distanciamiento social, lo que sin duda provocó un alto costo económico y social (Ashraf, 2020; Hevia y Neumeyer, 2020). En consecuencia, los estragos en la economía, provocados por el confinamiento, han promovido la caída del producto interno bruto (PIB) y contracción del mercado laboral (Kato y Lengyel, 2020).

En respuesta a lo anterior y con la finalidad de minimizar el impacto sobre la economía, diversos países han planteado estrategias para la reincorporación laboral de los trabajadores, de acuerdo con los síntomas presentados, tiempo de evolución, disponibilidad de pruebas de laboratorio y riesgo de exposición; tomando en cuenta la clínica y los recursos de diagnóstico para la detección de COVID-19, mediante la reacción en cadena de la polimerasa con transcriptasa inversa (RT-PCR, por sus siglas en inglés) y pruebas serológicas para detección de anticuerpos (CDC, 2020; Rueda-Garrido, 2020).

Por otra parte, de acuerdo a la OMS existen a nivel mundial un aproximado de 2 billones de adultos que presentan sobrepeso y la tercera parte de estos son obesos (OMS, 2014), atribuido entre otras razones, al desarrollo de la industria que ha generado que los procesos productivos dependan en mayor medida de las máquinas, disminuyendo el gasto energético y originando cambios en el estilo de vida (Wang y Lobstein, 2006).

En los últimos años en Perú, se han producido modificaciones en la alimentación, caracterizadas por incremento en el consumo de alimentos con elevado contenido de azúcar, sodio y grasa de tipo saturada. De la misma manera, se ha visto disminuida la actividad física con el consecuente sedentarismo, favoreciendo una alta incidencia de sobrepeso y obesidad con tendencia al incremento (Pajuelo, 2019). Algunos reportes señalan que uno de cada 3 profesionales presentan sobrepeso y uno de cada 13 obesidad (Barcones-Molero et al., 2018; Myers y Myers, 2004) y además han identificado la asociación entre el ausentismo laboral e índice de masa corporal (IMC), el cual condiciona aumento de la morbilidad asociada a enfermedades crónicas (Velasquez et al., 2017); originando a su vez, inconvenientes relacionados con el trabajo, pudiendo incluso llegar hasta el cese del mismo (Malinauskiene et al., 2011; Robroek et al., 2013; Mejía et al., 2020), tal 
y como refieren estudios que han señalado la asociación entre el IMC y ausentismo laboral, condicionando un aumento de la morbilidad asociada a enfermedades crónicas (Lehnert et al., 2014; Gajewski et al., 2015; Velásquez et al., 2017).

Del mismo modo, durante el confinamiento por COVID, la reducción de la actividad física y aumento del comportamiento sedente es una reacción inevitable del aislamiento social, que aunado a la obesidad y disminución de la capacidad pulmonar incrementan el riesgo de desarrollar un cuadro clínico grave asociado a COVID-19 e importantes implicaciones metabólicas en la salud (Celis et al., 2020).

Por lo anteriormente expuesto, se planteó como objetivo del presente estudio, evaluar el proceso de reincorporación laboral de trabajadores recuperados y con alteraciones persistentes de COVID-19 que se desempeñan en empresas de la industria metalmecánica de Lima, Perú. Asímismo, valorar el impacto sobre la productividad en las empresas donde realizan sus labores.

\section{Materiales y Métodos}

Se realizó un estudio epidemiológico descriptivo de corte transversal, evaluando el proceso reincorporación de trabajadores que se recuperaron de COVID-19 independientemente de la gravedad del cuadro clínico, así como los sujetos COVID persistentes, pertenecientes a 46 industrias metalmecánicas de la ciudad de Lima, Perú durante el tercer trimestre de 2020 hasta el primer trimestre 2021.

Para ello, se incluyeron trabajadores que desempeñan cargos de operadores de instalaciones de procesamiento y fabricación metalúrgica; código 812 del clasificador nacional de ocupaciones 2015 (8121 operadores de instalaciones de procesamiento de metales y 8122 operadores de máquinas pulidoras, galvanizadoras y recubridoras de metales); excluyendo sujetos con comorbilidad, como establece el Decreto Supremo No 083-2020-PCM, publicado el 10 de mayo de 2020, se ha extendido el estado de emergencia hasta el 24 de mayo de 2020.

\section{Proceso de Reincorporación laboral}

Para el proceso de reincorporación, se realizaron las evaluaciones médicas ocupacionales de reintegro laboral, considerando monitorear el estado de salud de aquellos trabajadores identificados, con posibilidad de requerir recomendación o atención médica, debido a su condición de salud, bien sea de manera temporal o definitiva. Los criterios técnicos se muestran en la tabla 1.

Tabla 1. Criterios de base para la reincorporación al trabajo con base en clínica, tiempo posterior a exposición, pruebas RT- PCR y COVID persistente

\begin{tabular}{|c|c|c|c|}
\hline Según clínica o síntomas & $\begin{array}{c}\text { Según tiempo posterior a prueba } \\
\text { PCR+ }\end{array}$ & $\begin{array}{c}\text { Según pruebas de detección RT- PCR } \\
\text { (criterio CDC) }\end{array}$ & COVID persistente \\
\hline $\begin{array}{l}\text { Persona con Covid- } 19 \text { Sintomático } \\
\text { Ausencia de síntomas por } 3 \text { días (fiebre, } \\
\text { tos, sin cansancio), y al menos haber } \\
\text { transcurrido } 14 \text { días desde inicio de } \\
\text { síntomas. } \\
\text { Para pacientes graves } 24 \text { días (de } \\
\text { acuerdo a su condición). }\end{array}$ & $\begin{array}{l}\text { Persona con Covid-19 Asintomático } \\
14 \text { días sin síntomas desde su prueba } \\
\text { PCR+. } \\
14 \text { días sin síntomas desde su contacto } \\
\text { con paciente sintomático o sospechoso } \\
\text { de Covid-19 }\end{array}$ & $\begin{array}{l}\text { RT-PCR positiva mayor a } 12 \text { semanas } \\
\text { Aunque el paciente ya esté sin síntomas. } \\
\text { Esto no significa que la persona tenga } \\
\text { capacidad de contagiar. } \\
\text { Tanto el paciente sintomático como el } \\
\text { asintomático deberá guiarse por clínica y } \\
\text { tiempo posterior a exposición. }\end{array}$ & $\begin{array}{l}\text { Síntomas persistentes por más de } 4 \\
\text { semanas } \\
\text { Más frecuentes son fatiga, dolor en } \\
\text { las articulaciones, tos y dificultad } \\
\text { para respirar }\end{array}$ \\
\hline
\end{tabular}

Previo al procedimiento de reincorporación se revisaron las demandas de los puestos de trabajo 8121 y 8122 de cada empresa, definiendo de esta manera, las demandas cognitivas, físicas y emocionales que se muestran en la tabla 2., como criterios para las decisiones administrativas de no levantar el alta médica, reincorporación con o sin adaptación de tareas, en consonancia con el sistema de vigilancia osteomuscular, auditivo, conservación de la voz y psicosocial, además de las pautas emitidas por la OMS para mitigar la propagación del SARS-CoV-2.

\section{Valoración del estado de salud}

Se realizó la valoración de reincorporación de los trabajadores (examen físico). Para ello, se aplicaron escalas de valoración en pacientes con COVID-19 recuperados y COVID persistente, tales como: Escala de fatiga de Borg basal o antes del ejercicio y final o después del ejercicio, escala de disnea modificada del Medical Research Council (mMRC) y la escala de ansiedad y depresión de Goldberg (EADG). A cada trabajador se le aplicó la escala de disnea de Borg (Borg, 1973) en reposo (percepción de disnea durante las actividades de la vida diaria) y posterior a la prueba de esfuerzo con caminata de 6 minutos; la escala de disnea del Medical Research Council 
(MRC) o escala de Fletcher de 1952 revisada por Schilling en 1955 (Fletcher, 1959) y la escala de ansiedad y depresión de Goldberg -EADG (Goldberg et al., 1988), constituida por las sub-escalas de ansiedad y de depresión.

Tabla 2. Demandas para reincorporación COVID-19 recuperado y COVID persistente en operadores de instalaciones de procesamiento y fabricación metalúrgica

\begin{tabular}{|c|c|c|c|}
\hline \multirow{2}{*}{ Puesto de trabajo } & \multicolumn{3}{|c|}{ Demandas } \\
\hline & Cognitivas & Físicas & Emocionales \\
\hline $\begin{array}{l}\text { 8121: Operadores de instalaciones de } \\
\text { procesamiento de metales }\end{array}$ & $\begin{array}{l}\text { tratamiento de datos complejos, entrada } \\
\text { de datos que requiere una gran } \\
\text { concentración, cambio rápido de tareas, } \\
\text { uso de múltiples sistemas, toma de } \\
\text { decisiones complejas, toma de } \\
\text { decisiones rápiday de alto riesgo, gestión } \\
\text { de múltiples partes interesadas o } \\
\text { informes }\end{array}$ & $\begin{array}{l}\text { Movimiento repetitivo, posturas } \\
\text { estáticas, }\end{array}$ & Necesidad de ser flexible y resiliente \\
\hline $\begin{array}{l}\text { 8122: Operadores de máquinas } \\
\text { pulidoras, galvanizadoras y recubridoras } \\
\text { de metales }\end{array}$ & $\begin{array}{l}\text { Uso de múltiples sistemas, toma de } \\
\text { decisiones rápida y de alto riesgo }\end{array}$ & $\begin{array}{l}\text { Movimiento repetitivo, posturas } \\
\text { estáticas, movimiento ocasional de } \\
\text { objetos engorrosos o pesados }\end{array}$ & $\begin{array}{l}\text { Potencial de agresión o violencia, } \\
\text { necesidad de ser flexible y resiliente }\end{array}$ \\
\hline
\end{tabular}

Los resultados obtenidos se tabularon en una base de datos en hoja de cálculo de excel, desarrollada por microsoft para Windows. Para el análisis de datos se empleó estadística descriptiva a través de medidas de tendencia central o medida de centralización.

\section{Resultados}

En la tabla 3., observamos que los recuperados son reincorporados a sus labores habituales por encima de $80 \%$ mientras que los COVID persistente alcanza cerca del 75\% para el cargo 8121 (Tabla 3). No obstante, este porcentaje de reincorporación disminuye para el cargo 8122, principalmente en los COVID persistente (43,33\%). Estas diferencias porcentuales son debidas a las demandas per se de los cargos, principalmente debidas al esfuerzo físico por movimiento repetitivo, posturas estáticas, movimiento ocasional de objetos engorrosos o pesados.

Tabla 3. Reincorporación porcentual de trabajadores recuperados COVID-19 y COVID persistente

\begin{tabular}{|c|c|c|c|c|c|c|c|c|c|c|c|c|}
\hline \multirow{4}{*}{ Puesto de trabajo } & \multicolumn{12}{|c|}{ \% de Reincorporación } \\
\hline & \multicolumn{9}{|c|}{ Recuperados COVID-19 } & \multirow{2}{*}{\multicolumn{3}{|c|}{ COVID persistente }} \\
\hline & \multicolumn{3}{|c|}{ Síntomas } & \multicolumn{3}{|c|}{ PCR+ } & \multicolumn{3}{|c|}{ n RT- PCR } & & & \\
\hline & $\mathrm{N}$ & $\mathrm{RI}$ & $\%$ & $N$ & $\mathrm{RI}$ & $\%$ & $\mathrm{~N}$ & $\mathrm{RI}$ & $\%$ & $N$ & $\mathrm{RI}$ & $\%$ \\
\hline 8121 & 152 & 125 & 82,24 & 96 & 79 & 82,29 & 87 & 75 & 86,21 & 51 & 38 & 74,51 \\
\hline 8122 & 651 & 397 & 60,98 & 154 & 98 & 63,64 & 149 & 89 & 59,73 & 150 & 65 & 43,33 \\
\hline Total & 803 & 522 & 65,01 & 250 & 177 & 70,80 & 236 & 164 & 69,49 & 201 & 103 & 51,24 \\
\hline
\end{tabular}

$\mathrm{N}$ número de evaluados, RI reincorporados

Otro aspecto a considerar desde el punto de vista organizacional, pero fundamentalmente financiero es el porcentaje de no reincorporación o de baja médica, en el caso de persistencias de síntomas que impidan al trabajador desarrollar sus labores o predispongan a accidente laboral, alcanza el 56,67\% de los individuos evaluados ocupantes del cargo 8122. Asimismo, 48,76\% del total general (cargos 8121 y 8122) COVID persistente, no estaba apto para ser reincorporado a sus labores habituales.

En la tabla 4., se observa que el promedio de la población trabajadora estudiada tiene medidas antropométricas acordes a los valores referenciales para Perú en hombres y mujeres para talla y peso, sin embargo, el IMC de la totalidad de la muestra presentó valores superiores a $26 \mathrm{Kg} / \mathrm{m}^{2}$; siendo el promedio más elevado en mujeres $\left(27,4 \mathrm{Kg} / \mathrm{m}^{2}\right)$ es decir, una masa corporal superior al valor referencial. Además, no hubo diferencias en el promedio de IMC entre trabajadores recuperados y COVID persistente. Por otra parte, aunque el 82,75\% $(n=1233)$ trabajadores reincorporados fueron del sexo masculino, el alta en trabajadores COVID persistente represento el 59,99\% de los casos.

En la figura 1., se muestra que en la totalidad de la población objeto a evaluación para retorno a la actividad laboral, las constantes fisiológicas Temperatura corporal, frecuencia cardíaca y frecuencia respiratoria se encuentran dentro de los parametros referenciales, es decir, no constituyen impedimento a considerar para una baja médica. 
Tabla 4. Antropométrica de los trabajadores recuperados COVID-19 y COVID persistente

\begin{tabular}{|c|c|c|c|c|c|c|c|c|c|c|c|c|c|c|c|c|}
\hline \multirow{5}{*}{$\begin{array}{c}\text { Datos } \\
\text { antropométricos }\end{array}$} & \multicolumn{16}{|c|}{ Operadores de instalaciones de procesamiento y fabricación metalúrgica } \\
\hline & \multicolumn{9}{|c|}{8121} & \multicolumn{7}{|c|}{8122} \\
\hline & \multicolumn{4}{|c|}{ Recuperados COVID-19 } & \multicolumn{4}{|c|}{ COVID persistente } & \multicolumn{4}{|c|}{ Recuperados COVID-19 } & \multicolumn{4}{|c|}{ COVID persistente } \\
\hline & \multicolumn{2}{|c|}{$\begin{array}{c}\text { Hombres } \\
\mathrm{N}=274\end{array}$} & \multicolumn{2}{|c|}{$\begin{array}{c}\text { Mujeres } \\
\mathrm{N}=61\end{array}$} & \multicolumn{2}{|c|}{$\begin{array}{c}\text { Hombres } \\
\mathrm{N}=14\end{array}$} & \multicolumn{2}{|c|}{$\begin{array}{c}\text { Mujeres } \\
\mathrm{N}=37\end{array}$} & \multicolumn{2}{|c|}{$\begin{array}{c}\text { Hombres } \\
\mathrm{N}=912\end{array}$} & \multicolumn{2}{|c|}{$\begin{array}{c}\text { Mujeres } \\
\mathrm{N}=42\end{array}$} & \multicolumn{2}{|c|}{$\begin{array}{c}\text { Hombres } \\
\mathrm{N}=33\end{array}$} & \multicolumn{2}{|c|}{$\begin{array}{c}\text { Mujeres } \\
\mathrm{N}=117\end{array}$} \\
\hline & Ц & $\sigma$ & Ц & $\sigma$ & Ц & $\sigma$ & Ц & $\sigma$ & Ц & $\sigma$ & ц & $\sigma$ & Ц & $\sigma$ & Ц & $\sigma$ \\
\hline Talla (M) & 1,64 & 0,23 & 1,52 & 0,21 & 1,63 & 0,42 & 1,54 & 0,14 & 1,64 & 0,47 & 1,52 & 0,48 & 1,63 & 0,22 & 1,51 & 0,51 \\
\hline Peso (Kg) & 70,25 & 1,42 & 66,27 & 1,45 & 69,52 & 2,54 & 62,25 & 1,68 & 70,25 & 6,25 & 64,23 & 1,98 & 69,52 & 1,11 & 64,20 & 3,21 \\
\hline IMC & 26,01 & 0,25 & 28,87 & 0,68 & 26,20 & 0,19 & 26,60 & 0,11 & 26,01 & 0,19 & 27,68 & 0,54 & 26,32 & 0,06 & 28,46 & 0,10 \\
\hline
\end{tabular}
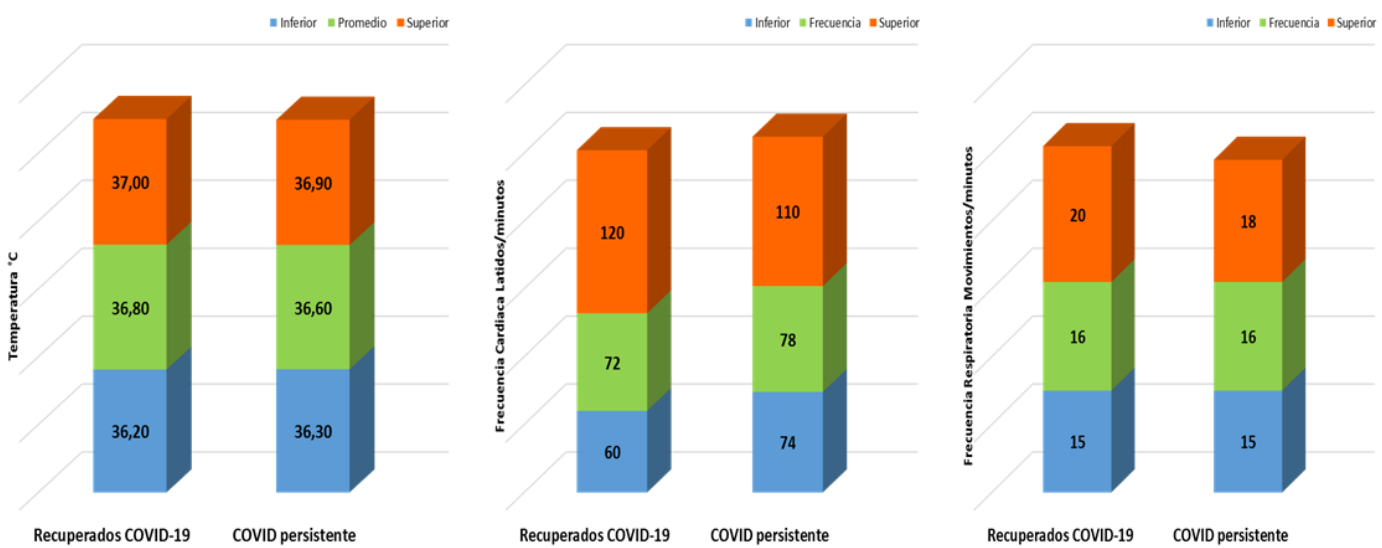

Figura 1. Constantes fisiologicas en trabajadores recuperados COVID-19 y COVID persistente

El esfuerzo respiratorio en reposo se encuentra entre los limites normales por la escala de Borg (Tabla 5), sin embargo, al realizar la prueba o ejercicio de la caminata en tiempo de 6 minuros la percepción de este esfuerzo pasa a grado de duro (Grado 5) en 13 y 85 trabajadores de categoria 8121 y 8122 respectivamente con COVID persistente, lo que indica la baja médica, con la consecuencia de $74,51 \%$ y $43,33 \%$ de no incorporación a la actividad laboral. Igual situación se presenta tras el ejercicio, pero en menor proporción, lo cual tiene la misma decisión administrativa.

Tabla 5. Valoración de la disnea en trabajadores COVID-19 recuperados y COVID persistente

\begin{tabular}{|c|c|c|c|c|c|c|c|c|c|c|c|c|c|c|c|c|}
\hline \multirow{3}{*}{ Escala } & \multicolumn{8}{|c|}{ Recuperados COVID-19 } & \multicolumn{8}{|c|}{ COVID persistente } \\
\hline & \multicolumn{4}{|c|}{ Hombres $\mathrm{N}=1233$} & \multicolumn{4}{|c|}{ Mujeres $\mathrm{N}=103$} & \multicolumn{4}{|c|}{ Hombres $=47$} & \multicolumn{4}{|c|}{ Mujeres $=154$} \\
\hline & $ц$ & $\sigma$ & $\min$ & Máx & $ц$ & $\sigma$ & $\min$ & Máx & Ц & $\sigma$ & $\min$ & Máx & $ц$ & $\sigma$ & $\min$ & Máx \\
\hline Borg Reposo & 0,92 & 1,24 & 0 & 3 & 1,08 & 1,43 & 0 & 4 & 0,87 & 1,27 & 0 & 4 & 1,45 & 0,56 & 0 & 4 \\
\hline Borg Ejercicio & 4,57 & 3,16 & 0 & 10 & 5,24 & 3,57 & 1 & 10 & 3,89 & 3,26 & 1 & 10 & 5,21 & 3,54 & 1 & 9 \\
\hline MRC & 2,05 & 1,83 & 0 & 4 & 3,25 & 4,57 & 1 & 4 & 2,56 & 0,98 & 0 & 4 & 2,73 & 2,29 & 0 & 4 \\
\hline
\end{tabular}

En recuperados COVID-19, se obtuvo un valor $\mathrm{R}^{2}=0.2986$ (Figura 2) lo que explica la respuesta de la variable disnea, como predictor al intensificar la actividad de rutina como vestirse y desvertirse, caminar en plano o en leves inclinaciones; por lo tanto, se ubican en los categorizados moderado y severo, pero no evidencian síntomas de dificultad respiratoria en la vida diaria; la orden administrativa es continuar con reposo domiciliario. El valor de $\mathrm{R}^{2}$ de 0.0501 (Figura 3) para COVID persistente. Todo trabajador que tenga compromiso respiratorio o 2 condiciones en la Escala de Goldberg -EADG se mantiene en baja por 14 días bajo observación médica. 


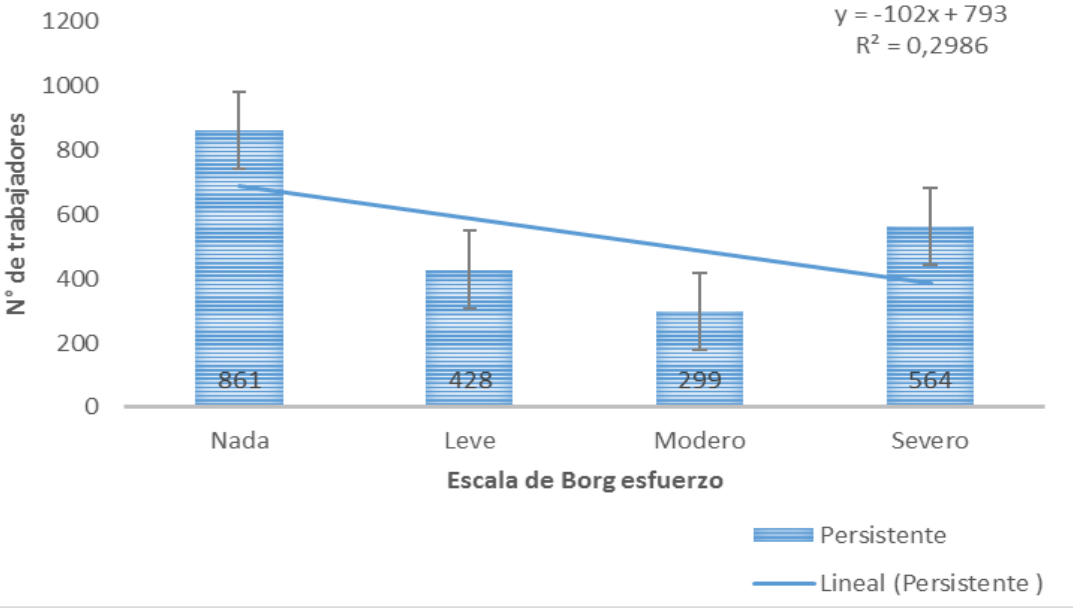

Figura 2. Categorización disnea percibida con esfuerzo en trabajadores COVID-19 recuperados

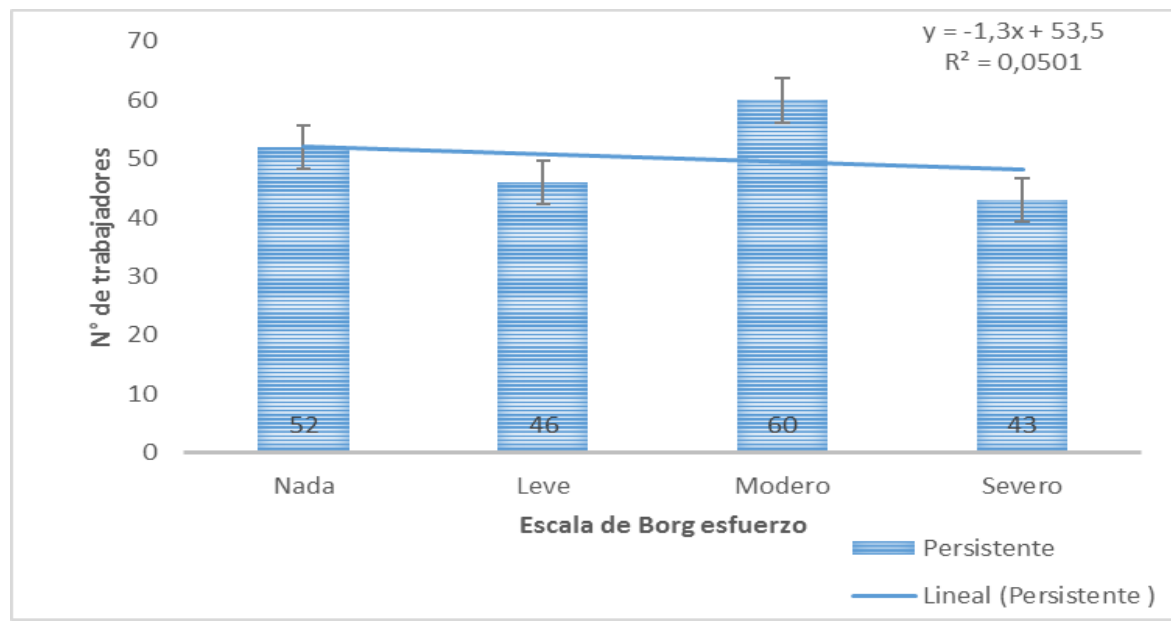

Figura 3. Categorización disnea percibida con esfuerzo en trabajadores COVID persistente

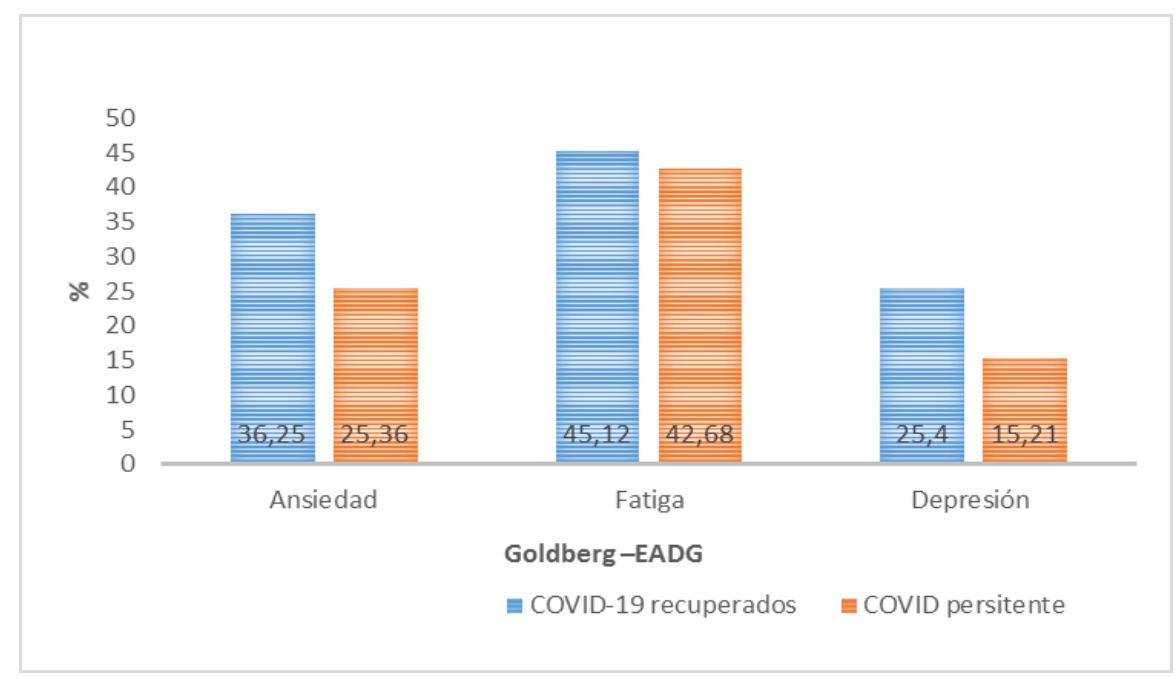

Figura 4. Escala de Goldberg -EADG en trabajadores COVID-19 recuperados y COVID persistente

\section{Discusión}

Los individuos con síndrome post-COVID o COVID persistente representan un desafío social, médico y laboral. Los resultados del presente estudio demostraron que los trabajadores con indicación de reincorporarse a sus actividades, 
mostraron al ingreso una "adecuada evaluación fisiológica", sin embargo, al aplicar los criterios de Borg, mMRC y Goldberg para su evaluación física; se encontró en un número considerable de trabajadores recuperados y COVID persistente, un conjunto de síntomas asociados a dicha patología, tales como; incremento del esfuerzo respiratorio post ejercicio y disnea post actividad física, en grado de moderado a severo.

Sánchez et al., (2021), en un estudio de metaanálisis indican que síntomas persistentes como tos, disnea y fatiga han sido observados comúnmente en pacientes post-COVID-19 entre 30 y 56 días post infección, no obstante, la evidencia indica que el COVID-19 puede tener efectos persistentes significativos hasta 12 semanas después del período de infección aguda, además existe una mayor prevalencia de fatiga en pacientes con COVID-19 grave y menor prevalencia de disnea (mMRC) en pacientes con antecedentes de enfermedad moderada a crítica. Por su parte Van Veenendaal, et al., (2021), en su estudio en pacientes COVID persistente, posterior a hospitalización en UCI, reportaron que $90 \%$ de los pacientes refirieron fatiga seis meses después del alta, mientras que, Carfi et al., (2020) y Halpin et al., (2021), informaron disnea en $42 \%$ y $66 \%$ respectivamente, en pacientes post infección por COVID-19; corroborando que los síntomas respiratorios se mantienen en un alto porcentaje de individuos, tal y como se observó en este estudio. En esta perspectiva, Sánchez et al., (2021) hacen referencia a un estudio que demuestra disminución de la capacidad funcional en pacientes post infección con un promedio de 4,2 meses de evolución. Asímismo, Huang et al., (2021), encontraron alteraciones en la prueba de marcha de 6 minutos, a los 6 meses post infección, similar a los resultados obtenidos según la escala de Borg, demostrando los efectos persistentes del COVID sobre la función respiratoria. No menos importante es lo referido por Sánchez et al., (2021) respecto a que alrededor de 6 semanas después de la aparición de los síntomas, 61,5\% de los pacientes retrasaron su regreso al trabajo al menos 5 semanas desde el inicio de la enfermedad siendo las principales causas fatiga y debilidad.

Por otra parte, resultados del presente estudio evidencian que tanto los trabajadores recuperados como los COVID persistente presentaron signos de ansiedad y/o depresión asociados a la enfermedad. En tal sentido Ozamiz et al., (2020), realizaron un análisis de los síntomas de estrés, ansiedad y depresión en individuos, con relación al COVID-19; encontrando alta prevalencia de problemas en la salud mental tales como: desánimo, negativismo, ansiedad crónica, angustia; asimismo, se demostró que $64 \%$ de la muestra que ha sufrido de ansiedad son mujeres en edades laboralmente productivas, el $58 \%$ ha presentado síntomas de estrés y $22 \%$ presentó síntomas crónicos de depresión. De igual manera, Van Veenendaal et al., (2021), evaluaron la ansiedad y depresión a los 3 y 6 meses post infección aguda en pacientes que estuvieron hospitalizados en UCI encontrando un funcionamiento psicológico "bueno" en las escalas de valoración. Cabezas y Sucuy (2020), al aplicar una prueba con escala DASS-21 en trabajadores de una empresa, identificaron que 66\%, 44\%, y 55\% presentaron depresión, estrés y ansiedad normal, respectivamente; constatando que existe una baja correlación del estado psicológico de los trabajadores y el COVID-19, no obstante, como se aprecia en el presente estudio son elementos que condicionan su salud mental, y por ende sus facultades para laborar de manera adecuada.

En particular, este estudio reveló que la totalidad de trabajadores recuperados y COVID persistente, presentaron sobrepeso al reintegrarse a sus actividades. El confinamiento ha conllevado a que gran parte de trabajadores hayan incrementado la ingesta de alimentos con patrones dietéticos poco saludables, con alto contenido en grasas y azúcares que aunado a la poca actividad física puede derivar en aumento de peso y mayor riesgo de obesidad, favoreciendo la aparición de diversos trastornos cardiometabólicos (Vásquez, 2021). Al respecto, Velásquez et al., (2017), demostraron que 49,4\% de trabajadores de una empresa en Perú presentaban sobrepeso y el 21,3\% de ellos se había ausentado de su trabajo, observando que conforme se incrementaba el IMC se hacían más frecuentes los grados de ausentismo a mediano y largo plazo. Igualmente, Gomero y Palomino (2012), hallaron asociación entre ausentismo laboral y el IMC en una empresa minera del Perú, demostrando que dicho trastorno repercute de manera negativa en la productividad y el estado financiero de las organizaciones.

Evidentemente, las personas moderada o extremadamente obesas a menudo tienen dificultad para moverse a causa de su tamaño corporal y al peso, por lo que están limitadas para realizar adecuadamente sus labores (Christensen et al., 2013); además, se ha descrito que presentan episodios constantes de dolor asociado a problemas musculoesqueléticos, dolor en articulaciones de los pies, las rodillas, los tobillos y la espalda (Gates et al, 2008). Del mismo modo, el sobrepeso y obesidad repercuten sobre la función respiratoria y el intercambio de gases; los pacientes obesos presentan una respiración más rápida y superficial (en comparación con los normopeso) para adaptarse al incremento del tejido adiposo en la pared torácica, siendo de mal pronóstico en enfermedades como asma, síndrome de obesidad-hipoventilación y neumonía entre otras. Adicionalmente, el estado inflamatorio crónico asociado al exceso de peso suele potenciar la respuesta inmunitaria contra la infección viral, lo que resulta en autolesión de los pulmones y una evolución mucho más grave, tal y como se aprecia en el COVID, el cual se asocia a incremento de los niveles séricos de citocinas proinflamatorias que median la gravedad de la enfermedad (Echevarría, 2020).

Como puede apreciarse, el COVID-19 además de afectar la salud de trabajadores (recuperados y COVID persistentes), afecta también la productividad y economía de las empresas donde estos realizan sus actividades, originando disminución en el reintegro al trabajo, incremento de permisos de salud (por disfunción laboral tanto física como psicológica 
relacionada con los síntomas) y, en consecuencia, altas tasas de desempleo, generando a su vez mayor depresión y ansiedad entre los trabajadores. En ese sentido, es necesario aplicar estrategias similares a las adoptadas para otras afecciones, que promuevan el reintegro seguro al trabajo, como las referidas por Godeau et al., (2021), quienes recomiendan el uso de escalas como el Índice de capacidad laboral (WAI) o Impedimento de la productividad y la actividad laboral (WPAI), lo que permitiría un seguimiento a largo plazo y proporcionar información sobre la capacidad y carga de trabajo.

\section{Agradecimientos}

A nuestras casas de estudio, cuna de saberes.

\section{Conflicto de intereses}

Ninguno que declarar.

\section{Referencias}

Aiyegbusi, O.L., Hughes, S.E., Turner, G., Rivera, S.C., McMullan, C., Chandan, J.S., Haroon, S., Price, G., Davies, E.H., Nirantharakumar, K., Sapey, E. \& Calvert, M.J. (2021). Symptoms, complications and management of long COVID: a review. J R Soc Med. Jul 15:1410768211032850. https://doi/10.1177/01410768211032850.

Ashraf, B. N. (2020). Economic impact of government interventions during the COVID-19 pandemic: International evidence from financial markets. Journal of Behavioral and Experimental Finance, 27, 1-9. https://doi.org/10.1016/j.jbef.2020.100371

Barcones-Molero, M.F., Sánchez-Villegas, A., Martínez-González, M.A., Bes-Rastrollo, M., Martínez-Urbistondo, M. \& Santabárbara, J. (2018), Influencia de la obesidad y la ganancia de peso sobre la calidad de vida según el SF- 36 en individuos de la cohorte dinámica Seguimiento Universidad de Navarra. Rev Clínica Esp. 218(8):408-16. https://doi.org/10.1016/j. rce.2018.05.005

Borg, G. (1976). Simple rating methods for estimation of perceived exertion. Wenner-Gren Center International Symposium. Series 28:39-47.

Cabezas, E. \& Sucuy, I. (2020). Afrontamiento del COVID-19 y bioseguridad laboral en la Empresa SEMAICA. Ecuador. Disponible en; http://dspace.unach.edu.ec/handle/51000/7026. (Acceso junio 2021)

Carfi, A., Bernabei, R., Landi, F., Gemelli \& COVID-19 Post-Acute Care Study Group. (2020). Persistent Symptoms in Patients After Acute COVID-19 JAMA. Aug 11;324(6):603-605. https://doi.org/10.1001/jama.2020.12603

Celis, C., Salas, C., Yáñez, A. \& Castillo, M. (2020). Inactividad física y sedentarismo. La otra cara de los efectos secundarios de la Pandemia de COVID-19 Revista médica de Chile. 148 (6), 885886. https://dx.doi.org/10.4067/S0034-98872020000600885

Center for Disease Control and Prevention. Criteria for Return to Work for Healthcare Personnel with Suspected or Confirmed COVID-19 (Interim Guidance). (2020). Disponible en: https://www.cdc.gov/coronavirus/2019ncov/hcp/return-to-work.html (Acceso junio 2021)

Christensen, J., Overgaard, K., Hansen, K., Søgaard, K. \& Holtermann, A. (2013). Effects on presenteeism and absenteeism from a 1-year workplace randomized controlled trial among health care workers. J Occup Environ Med. Oct;55(10):1186-90. https://doi.org/10.1097/JOM.0b013e31829b2816

Echevarría, D. (2020). La obesidad: posibles mecanismos que explican su papel como factor de riesgo de la COVID-19 Revista Cubana de Alimentación y Nutrición. 30:(1) (Suppl 2): S30-S41. Disponible en; http://www.revalnutricion.sld.cu/index.php/rcan/article/view/988/pdf 229 . (Acceso junio 2021)

Fletcher, C.M., Elmes, P.C. \& Wood, C.H. (1959). The significance of respiratory symptoms and the diagnosis of chromic bronchitis in a working population. B.M.J. 1:257-266. https://doi.org/10.1136/bmj.2.5147.257

Gajewski, K., Burris, D., Spears, D.R., Sullivan, K., Oyinloye, O. \& McNeil, C. (2015). Demographic trends of sick leave absenteeism among civil service employees at a Federal Agency from 2004 to 2012. JOEM. 57(3):277-83. https://doi.org/10.1097/JOM.0000000000000347

Gates, D. M., Succop, P., Brehm, B. J., Gillespie, G. L., \& Sommers, B. D. (2008). Obesity and presenteeism: the impact of body mass index on workplace productivity. Journal of occupational and environmental medicine, 50(1): 3945. https://doi.org/10.1097/JOM.0b013e31815d8db2 
Godeau, D., Petit, A., Richard, I., Roquelaure, Y., \& Descatha, A. (2021). Return-to-work, disabilities and occupational health in the age of COVID-19. Scandinavian journal of work, environment \& health, 47(5): 408-409. https://doi.org/10.5271/sjweh.3960

Goldberg, D., Bridges, K., Duncan-Jones, P., \& Grayson, D. (1988). Detecting anxiety and depression in general medical settings. BMJ (Clinical research ed.), 297(6653): 897-899. https://doi.org/10.1136/bmj.297.6653.897

Gomero, R. \& Palomino, J. (2012). Absentismo laboral e índice de masa corporal. Revista de Seguridad Minera. (58):267. Disponible en: https://www.revistaseguridadminera.com/salud-ocupacional/absentismo-laboral-e-indice-demasa-corporal/ (Acceso septiembre 2021).

Halpin, S. J., McIvor, C., Whyatt, G., Adams, A., Harvey, O., McLean, L., Walshaw, C., Kemp, S., Corrado, J., Singh, R., Collins, T., O'Connor, R. J., \& Sivan, M. (2021). Postdischarge symptoms and rehabilitation needs in survivors of COVID-19 infection: A cross-sectional evaluation. Journal of medical virology, 93(2):1013-1022. https://doi.org/10.1002/jmv.26368

Hevia, C. \& Neumeyer, A. (2020). Un marco conceptual para analizar el impacto económico del COVID-19 y sus repercusiones en las políticas. Serie de Documentos de Política Pública. Disponible en: https://www.latinamerica.undp.org/content/rblac/es/home/library/crisis_prevention_and_recovery/a-conceptualframework-for-analyzing-the-economic-impact-of-covi.html (Acceso junio 2021)

Huang, C., Huang, L., Wang, Y., Li, X., Ren, L., Gu, X., Kang, L., Guo, L., Liu, M., Zhou, X., Luo, J., Huang, Z., Tu, S., Zhao, Y., Chen, L., Xu, D., Li, Y., Li, C., Peng, L., Li, Y., Xie, W.,, Cui D., Shang, L., Fan, G., Xu, J., Wang, G., Wang, Y., Zhong, J., Wang, C., Wang, J., Zhang, D. \& Cao, B. (2021). 6-month consequences of COVID-19 in patients discharged from hospital: a cohort study. Lancet (London, England), 397(10270), 220-232. https://doi.org/10.1016/S0140-6736(20)32656-8

Kato, E. \& Lengyel K. E. (2020). De lo perdido y recuperado: severidad de recesión y crecimiento. En R. Pineda López, M.T. García Gasca, A. Ochoa Cervantes, J.A. Hernández Guerrero (Eds.). Análisis y perspectivas sobre la pandemia, de COVID-19 en Querétaro (pp. 346-376). México: Universidad Autónoma de Querétaro Lehnert, T., Stuhldreher, N., Streltchenia, P. (2014). Sick leave days and costs associated with overweight and obesity in Germany. J Occup Environ Med. 56(1):20-7. https://doi.org/10.1097/JOM.0000000000000065

Macdonald, E., Lalloo, D., Raynor, C, \& Yarker, J. (2021). Reincorporación al trabajo después de la infección por COVID19 y covid persistente - Guía para los trabajadores. Long Covid Group Agencia Europea para la Seguridad y la Salud en el Trabajo (Society of Occupational Medicine). Disponible en: https://osha.europa.eu/es . (Acceso junio 2021).

Malinauskiene, V., Leisyte, P., Romualdas, M. \& Kirtiklyte, K. (2011). Associations between self-rated health and psychosocial conditions, lifestyle factors and health resources among hospital nurses in Lithuania. J Adv Nurs. 67(11):2383-2393. https://doi.org/10.1111/j.1365-2648.2011.05685.x

Mejia, C., Llontop-Ramos, F., Vera, C., Caceres, O. (2020). Factores asociados a sobrepeso y obesidad en trabajadores de Piura-Perú. Rev. Fac. Med. Hum. 20(3):351-357. https://doi.org/10.25176/RFMH.v20i3.2904

Myers, H. L., \& Myers, L. B. (2004). 'It's difficult being a dentist': stress and health in the general dental practitioner. British dental journal. 197(2):89-101. https://doi.org/10.1038/sj.bdj.4811476

Ozamiz-Etxebarria, N., Dosil-Santamaria, M., Picaza-Gorrochategui, M. \& Idoiaga-Mondragon, N. (2020). Stress, anxiety, and depression levels in the initial stage of the COVID-19 outbreak in a population sample in the northern Spain. Cad Saude Publica. Apr 30;36(4):e00054020. https://doi.org/0.1590/0102-311X00054020

Pajuelo, J., Torres, L., Agüero, R. \& Bernui, I. (2019). El sobrepeso, la obesidad y la obesidad abdominal en la población adulta del Perú. Anales de la Facultad de Medicina, 80(1), 21-27. https://dx.doi.org/10.15381/anales.v80i1.15863

Riesco, J., de la Rosa, D. \& Jiménez, C. (2021). $2^{\circ}$ Congreso Nacional Multidisciplinar Covid-19 de las Sociedades Científicas de España. Disponible en: https://2congresocovid.es/2congresocovid (Acceso junio 2021)

Robroek, S.J., Schuring, M., Croezen, S., Stattin, M. \& Burdorf, A. (2013). Poor health, unhealthy behaviors, and unfavorable work characteristics influence pathways of exit from paid employment among older workers in Europe: a four year follow-up study. Scand J Work Environ Health. 125-133. https://dx.doi.org/10.5271/sjweh.3319 
Rueda-Garrido, J., Vicente-Herrero, M., del Campo, M., Reinoso-Barbero, L., de la Hoz, R., Delclos, G., Kales, S. \& Fernández-Montero, A. (2020). Return to work guidelines for the COVID-19 pandemic. Occup Med (Lond). Jun 1:6. https://doi:10.1093/occmed/kqaa099

Sánchez, D., Normand, K., Zhaoyun, Y. \& Torres, R. (2021). Long-Term Impact of COVID-19: A Systematic Review of the Literature and Meta-Analysis. Biomedicines. Jul 27;9(8):900. https://doi.org/10.3390/biomedicines9080900

Sociedad Española de Médicos Generales y de Familia (SEMG). (2020). COVID-19 persistente. Disponible en: https://www.semg.es/images/2020/Noticias/20201111_Resultados_Encuesta_COVID_Persistente.pdf (Acceso junio 2021).

Van Veenendaal, N., van der Meulen, I.C., Onrust, M., Paans, W., Dieperink, W. \& van der Voort P,H,J. (2021). Six-Month Outcomes in COVID-19 ICU Patients and Their Family Members: A Prospective Cohort Study. Healthcare (Basel). Jul 8;9(7):865. https://doi/0.3390/healthcare9070865

Vásquez, D. (2021). Revisión crítica: efecto del confinamiento por covid-19 en la ganancia de peso en el adulto. Disponible en; http://repositorio.uwiener.edu.pe/bitstream/handle/123456789/4881/T061_17858935_S.pdf?sequence=1\&isAllo wed $=\mathrm{y}$ (Acceso junio 2021).

Velásquez, C., Palomino, J. \& Ticse, R. (2017). Relación entre el estado nutricional y los grados de ausentismo laboral en trabajadores de dos empresas peruanas. Acta Médica Peruana, 34(1), 6-15. Disponible en: http://www.scielo.org.pe/scielo.php?script=sci_arttext\&pid=S1728-59172017000100002\&lng=es\&tlng=es (Acceso junio 2021).

Wang. Y. \& Lobstein. T. Worldwide trends in childhood overweight and obesity. (2006). Int J Pediatr Obes. 1(1):11-25. https://doi/10.1016/S0140-6736(17)32129-3

World Health Organization (WHO). (2014). World Heath Statistics. Disponible en: http://apps.who.int/iris/bitstream/handle/10665/112738/9789240692671_eng.pdf?sequence=1 (Acceso junio 2021). 